\title{
NUEVAS FUENTES PARA EL ANÁLISIS DEL TRANSPORTE INTERNACIONAL DE MERCANCÍAS A TRAVÉS DE LOS PIRINEOS
}

\author{
Ana Isabel ESCALONA y Víctor ANDRÉS \\ Departamento de Geografía y Ordenación del Territorio \\ Universidad de Zaragoza
}

\begin{abstract}
Resumen: La supresión de aduanas en la frontera hispano francesa supuso la interrupción de series estadísticas de enorme interés para estimar la evolución del tráfico de mercancías a través de los Pirineos así como su reparto entre las distintas travesías. En este orden de cosas el artículo presenta una fuente relativamente nueva, como es el fichero con datos de paso de vehículos por fronteras que desde 1995 gestiona la Dirección General de Tráfico. Un primer análisis de tales datos confirma, en opinión de los autores, el carácter "insostenible" del patrón de circulación vigente y la necesidad de que se promuevan alternativas tanto modales, a favor del transporte ferroviario, como en rutas, por el centro de la cadena.
\end{abstract}

Palabras clave: Modelo de tráfico de mercancías, Pirineos.

Abstract:: This article introduces a new source for the analysis of commoditys transport across the Pyrenees, which is the data base of the Direccion General de Tráfico, concerning entries and exits of vehicles through 13 border points between France and Spain. The results for 1997 show that current traffic pattern is reaching insostenibility and that alternative patterns, relying on railways and through the central section of Pyrenees, must be promoted.

Key Words: Current traffic pattern, Pyrenees.

\section{INTRODUCCIÓN}

La entrada en vigor del Mercado Unico europeo en enero de 1993 tuvo, entre otros efectos, el de la supresión de las aduanas situadas en la frontera hispano francesa y, en consecuencia, la interrupción de series estadísticas de enorme interés para estimar la evolución del tráfico de mercancias a través de los Pirineos así como su reparto entre las distintas carreteras, autopistas o ferrocarriles transpirenaicos. Efectivamente 
dichas estadísticas se basaban en el registro en cada aduana de los movimientos de mercancías, señalándose no sólo origen o destino, sino también posición de arancel, volumen y valor. Su localización, por último, en las principales carreteras y ferrocarriles que atraviesan los Pirineos, hizo de dichas estadísticas una fuente esencial para evidenciar algunas de las características de la circulación transpirenaica: su crecimiento constante así como su fuerte concentración espacial y modal.

La falta de esos datos desagregados por aduanas/vías de entrada o salida $h$ a privado a los investigadores y planificadores de una fuente esencial, justo en un momento en el que las caracteríticas e intensidad del tráfico, y sus secuelas, merecen en diversos foros el calificativo de "insostenibles" y suscitan una demanda creciente de reorganización general de la circulación transpirenaica, en la que se contemplen alternativas tanto modales, a favor del transporte ferroviario, como en rutas, por el centro de la cadena ${ }^{1}$. Resulta obvio el interés que para las regiones centropirenaicas como Aragón tienen tales propuestas.

El vacío informativo dejado por las estadísticas aduaneras puede ser paliado, hasta cierto punto, por otro tipo de datos como son los de tráfico transpirenaico de vehículos pesados. En este orden de cosas el objetivo del artículo es presentar una fuente relativamente nueva, como es el fichero generado desde 1995 a partir de los registros de un sistema electrónico implantado en los 13 pasos fronterizos principales, y cuya instalación y mantenimiento dependen de la Dirección General de Tráfico. Por falta de espacio nos limitaremos a presentar los datos de tráfico de vehículos pesados durante 1997, precedidos de una referencia a sus dos factores explicativos esenciales, como son la evolución reciente del comercio entre España, Portugal y los demás países de la Unión Europea; y la posición dominante que mantiene, y presumiblemente mantendrá, la carretera en la realización de dicho comercio. $Y$ todo ello con el ánimo de brindar soporte a los argumentos de quienes postulan que la reorganización de 1 a circulación transpirenaica de mercancías no puede esperar más ${ }^{2}$.

1 Como ejemplo reciente de ese interés mencionaremos el Simposio internacional "Transporte sostenible en zonas de montaña: El papel del ferrocarril en el transporte de mercancías por el Pirineo Central", celebrado en Jaca en los primeros días de octubre de 1998. Las consideraciones que siguen proceden en parte de la ponencia que presentamos a dicho simposio, titulada "La red de travesías pirenaicas y la evolución de los transportes" y que está pendiente de una pronta publicación.

2 Los datos que aparecerán a lo largo del texto proceden de los siguientes organismos e instituciones: Servicio de Documentación Territorial de la Diputación General de Aragón, Centro de Documentación Europea de la Universidad de Zaragoza, Fundación Ecología y Desarrollo, Centro de Documentación del Transporte y las Comunicaciones, "Data shop" Eurostat de Madrid, Unidad de Cargas de Renfe, Transfesa, Subdirección General de Planificación Estadística en el Departamento de Aduanas e Impuestos Especiales, Instituto de Estudios Turísticos, Centro de Control de Tráfico de la Dirección General de Tráfico, Jefatura Provincial de Tráfico de Zaragoza, Dirección general de Carreteras de la Diputación General de Aragón, Direction 


\section{UNA APROXIMACIÓN AL TRANSPORTE TRANSPIRENAICO DE MERCANCÍAS: EL COMERCIO DE ESPAÑA Y PORTUGAL CON LOS DEMÁS ESTADOS MIEMBROS DE LA UNIÓN EUROPEA}

Es cierto que, por su posición geográfica, los Pirineos son también paso obligado para las corrientes de mercancías entre los países del norte de Africa y la Europa extraibérica. Las previsiones del mismo apuntan, además, a un crecimiento sostenido. No obstante, por ahora, la parte sustancial del tráfico de mercancías es la que tiene como origen o destino España y Portugal. Lo que queremos poner de manifiesto es que la notable dinámica de tales intercambios representa un factor explicativo de primer orden de la intensidad alcanzada por el el tránsito transpirenaico de mercancías, que supera al de los Alpes franceses ${ }^{3}$ y cuyas expectativas de crecimiento van a mantenerse en los próximos años.

El punto de partida es lo que ha supuesto para ambos países su incorporación a la Europa comunitaria, y la posterior entrada en vigor del Mercado Unico, en el sentido de incrementar sus transacciones comerciales con los nuevos socios en perjuicio del comercio efectuado con el resto del mundo ${ }^{4}$. Ello ha acarreado un aumento de los transportes de mercancías a través de los Pirineos, con especial énfasis en las relaciones norte-sur, ya que es en las importaciones donde se observan los crecimientos más generalizados en los dos países. La excepción más notable se da en el comercio con Alemania, donde los flujos de exportación sur-norte son los que más han crecido. Por otra parte, en el patrón geográfico de los intercambios resalta la alta concentración de compras y ventas en un reducido número de países, que vienen a coincidir para España y Portugal. De hecho en 1996 el grupo formado por Francia, Alemania, Reino Unido e Italia representaban el destino del $50 \%$ de las exportaciones totales portuguesas, así como el origen del $44,1 \%$ de las importaciones. Por lo que respecta a España los cuatro países antes citados también son en 1996 origen y destino preferentes: del 50,5\% de las importaciones y del 51 ' $8 \%$ de las exportaciones totales.

\footnotetext{
Régionale de l'Equipement de Midi-Pyrénees. A las personas que allí nos atendieron vaya nuestro sincero agradecimiento.

3 Véase al respecto Etchélecou, A., Axe Pau-Somport. Quel trafic de poids lourds? Le prévu et le probable. IRSAM, Universidad de Pau, 1995.

${ }^{4}$ En el caso de España, y como se deduce de los datos de Eurostat, las exportaciones a los países ultrapirenaicos han pasado de representar el 59,3\% del total en 1986 al 62,8 en 1996. Los datos son más expresivos aún en lo que se refiere a las importaciones de esa área económica: $52,3 \%$ del total en 1986 y 63,4\% en 1996. Por lo que respecta a Portugal la variación observada es menos espectacular, dado que en los cálculos no se han incluído los intercambios con uno de sus principales socios, España, ya que no pasan por los Pirineos. Así en 1986 eran ultrapirenaicas el $70 \%$ de las exportaciones y el 51,1\% de las importaciones totales de ese país, mientras que en 1996 tales exportaciones se situan en el $65,4 \%$ y las importaciones en el $53,6 \%$.
} 
El tiempo dirá si esa concentración geográfica se ve alterada por intercambios con otros destinos o procedencias, máxime con las perspectivas de incorporación a 1 a Unión Europea de nuevos países. También habrá que contar con la posibilidad de que, dada la notable concentración sectorial del comercio con el grupo de países citado -el $50 \%$ de las exportaciones, por ejemplo, está compuesto por el sector agroalimentario y el del automóvil-, pueda verse afectado por alteraciones en la evolución cíclica de algunos productos, o la variabilidad de la oferta en el caso de productos agrarios ${ }^{5}$. Pero lo que los expertos parecen tener claro es que el volumen de los intercambios desde/hacia España y Portugal, y por tanto el tráfico de mercancías por los Pirineos, se mantendrá como mínimo, toda vez que las economías española y portuguesa han alcanzado niveles de apertura e internacionalización semejantes a los de otros países europeos.

Estamos pués ante un proceso de carácter estructural en el que la actividad exportadora está dejando de ser algo marginal para convertirse en objetivo prioritario de una parte creciente de las empresas de ambos países ${ }^{6}$.

En última instancia habría además otros factores que apuntan en la misma dirección de incremento del tránsito de mercancías a través de los Pirineos, como son 1 a influencia del Mercado Unico europeo en la la preponderancia de los intercambios intracomunitarios de todos los estados miembros, y el hecho de que "una parte sustancial de este comercio está representada por flujos intraempresas, engendrados por el desarollo conjunto de la especialización, la constitución de redes económicas entre sociedades, la división geográfica del trabajo y la expansión territorial de los mercados". La cita proviene del Documento de Noordwijk, primer proyecto oficial de lo que será en su día el "Esquema de desarrollo del espacio comunitario" europeo7. Y efectivamente, como han reflejados investigaciones recientes, el perfil sectorial de las exportaciones e importaciones españolas denota ya un importante crecimiento del comercio intraindustrial, especialmente en las semimanufacturas (productos siderúrgicos y metales no ferrosos), bienes de equipo (material de transporte y maquinaria mecánica) y en el sector del automóvil ${ }^{8}$.

\footnotetext{
${ }^{5}$ Hernando Moreno, J.M., 1997, "El comercio exterior español: balance de una década en la Unión Europea". Información Comercial Española, $\mathrm{n}^{\circ} 766$.

6 Así opina, entre otros, Verdeguer Puig, en "El sector exterior: ¿la confirmación de un cambio estructural?". Economistas, n 77 (extra), 1998: 120-128.

7 La versión que hemos manejado fue presentada en la reunión informal de los ministros responsables de la ordenación del territorio de los estados miembros de la Unión Europea, celebrada en Noordwijk, Países bajos, en junio de 1997.

${ }^{8}$ Hernando Moreno, op. cit, pág. 156.
} 


\section{EL CRECIENTE DESEQUILIBRIO INTERMODAL DEL TRANSPORTE DE MERCANCÍAS A TRAVÉS DE LOS PIRINEOS}

La insostenibilidad del actual patrón de circulación de mercancías a través de los Pirineos no deriva en realidad de las perspectivas de crecimiento del comercio que acaban de señalarse, sino del hecho de que sea la carretera el modo mediante el que se efectua la mayoría de ese intenso y creciente intercambio comercial. No es algo nuevo ya que desde hace varios quinquenios este modo viene consolidando su posición dominante en el transporte internacional de mercancías por vía terrestre desde/hacia España y Portugal. Los datos de Eurostat para 1994 así lo confirman al reseñar que por carretera se efectua ya el 96,6 y el 92,2\% de dicho transporte en Portugal y España respectivamente 9 . De ahí el volumen de mercancías tan diferenciado que ha venido transitando por las aduanas pirenaicas de ferrocarril (Port-Bou e Irún) y por las de carretera (La Junquera e Irún TIR), tal como quedaba recogido en las desaparecidas estadísticas aduaneras ${ }^{10}$.

\section{El transporte transpirenaico de mercancías por ferrocarril}

Es un hecho que la proporción del ferrocarril en el transporte transpirenaico de mercancías ha ido en descenso ${ }^{11}$, aunque los datos que sustentan esa afirmación deben ser manejados con cautela tal y como ha advertido recientemente la oficina estadística de la Unión Europea ${ }^{12}$.

9 Eurostat, Anuario 97. Visión estadística sobre Europa 1986-1996. Luxemburgo, 1998.

${ }^{10}$ En nuestra tesis doctoral ya aportábamos detalles del despacho de mercancías por las aduanas pirenaicas en 1986 y 1987 (Véase Escalona Orcao, Ana Isabel, "Las comunicaciones pirenaicas en Aragón", Huesca, Instituto de Estudios Altoaragoneses, 1990). A título de ejemplo señalaremos los datos correspondientes a 1987, recién ampliada la Comunidad europea, cuando las importaciones despachadas en la aduana de Port-Bou ascendieron a 203.036 toneladas frente a los 3.150 .455 de La Junquera, mientras que las exportaciones sumaron 182.451 y 3.145 .232 toneladas también respectivamente. Y lo mismo puede decirse del otro extremo de la cadena pirenaica cotejando los tonelajes despachados en la aduanas TIR y ferrocarril de Irún. Este caso la aduana TIR despachó 2.481.169 toneladas a la importación frente a los 486.570 de su homóloga de ferrocarril. A la exportación fueron 2.103.275 toneladas frente a los 133.946 despachadas en la aduana de ferrocarril.

${ }^{11}$ En 1994 sólo se realizaba por ferrocarril el 3,4\% del transporte internacional de mercancías de Portugal y el $7,8 \%$ del de España

12 Efectivamente la mala calidad de la información estadística sobre transporte internacional de mercancías, y en especial por ferrocarril, es un problema generalizado para toda la Unión Europea, hasta el punto de que se considere como poco realistas los datos recogidos durante años en la publicación de Eurostat "El comercio exterior por modos de transporte". Los expertos aducen problemas en la clasificación por capítulos de las mercancías -donde se han constatado diferencias sustanciales de interpretación entre unos países y otros-. De ahí resultan notables desviaciones entre los tonelajes declarados por el país emisor y el receptor; desviaciones que pueden superar el $30 \%$ cuando se trata de cantidades anuales inferiores a 500.000 toneladas como ocurre en muchos de los movimientos feroviarios hacia/desde España y Portugal. En estos casos los expertos desaconsejan la utilización de tales estadísticas por su carácter poco realista (Eurostat, Report on 
Si nos referimos a la evolución del tráfico de cargas, la cuantía de las expediciones desde/hacia España refleja para todas las relaciones volúmenes muy por debajo de las que se encaminan por carretera y que después se verán (Tabla 1).

Tabla 1.- Volumen de las importaciones y exportaciones ferroviarias de España a través de los Pirineos (miles de toneladas).

\begin{tabular}{|lrrrr|}
\hline & \multicolumn{2}{c}{ Importaciones } & \multicolumn{2}{c|}{ Exportaciones } \\
Origen/Destino & 1995 & 1996 & 1995 & 1996 \\
\hline Bélgica & 55 & 56 & 5 & 4 \\
Dinamarca & 0 & 0 & 0 & 1 \\
Alemania & 201 & 255 & 127 & 225 \\
Grecia & 0 & 0 & 0 & 0 \\
Francia & 575 & 548 & 667 & 565 \\
Irlanda & 0 & 0 & 0 & 0 \\
Italia & 27 & 37 & 42 & 97 \\
Luxemburgo & 21 & 17 & 0 & 0 \\
Holanda & 9 & 15 & 0 & 1 \\
Reino Unido & 0 & 0 & 0 & 0 \\
Finlandia & 0 & 0 & 0 & 0 \\
Suecia & 2 & 2 & 0 & 2 \\
Noruega & 0 & 0 & 0 & 0 \\
Austria & 27 & 41 & 0 & 6 \\
\hline Total & 917 & 971 & 841 & 901 \\
\hline
\end{tabular}

Fuente: Renfe, Unidad de cargas. Elaboración propia.

Resalta en estos datos la fuerte concentración de los tráficos en las relaciones con Francia y Alemania y, en mucha menor medida, con Italia. No obstante, dada la evolución general de este modo de transporte, el análisis no puede limitarse al seguimiento de su cuota de mercado respecto de otros, ni a la referencia exclusiva a l transporte de carga, sino que debe reseñar también la evolución en el transporte intermodal así como de algunos tráficos específicos (automóviles, determinados productos alimentarios, etc), en los que el ferrocarril presenta ventajas competitivas y, de hecho, incrementos del tráfico. A este respecto un rápido examen del último informe publicado por la empresa Transfesa proporciona diversos ejemplos de tráficos internacionales crecientes entre España y diversos países no sólo de la Europa

quality control of railway statistics in the European Union. Encuentro del grupo de trabajo europeo sobre estadísticas de transporte por ferrocarril celebrado en Luxemburgo 17 y 18 de noviembre de 1997. Documento T9/97/5). 
Occidental sino de Europa del Este y Escandinavia. También destacan para este tipo de transporte las relaciones con el Reino Unido, impulsadas tras la apertura del Eurotúnel. En su mayoría corresponden a piezas y componentes de automóvil, automóviles nuevos y productos químicos y graneles ${ }^{13}$.

\section{El transporte transpirenaico de mercancías por carretera}

En cualquier caso, y como decíamos al principio, hoy por hoy es la carretera el modo de transporte dominante de las mercancías que atraviesan los Pirineos y en el periodo 86-95 el tonelaje transportado entre España y Portugal y sus socios ultrapirenaicos ha aumentado de forma generalizada cuando no espectacular (Tablas 2 y 3$)^{14}$ La misma fuente proporciona información sobre los tipos de mercancías transportadas hacia/desde cada país en 1995 (Tabla 4). Fn la línea de las consideraciones hechas anteriormente sobre el comercio exterior, tanto en la importación como en la exportación destaca un reducido número de sectores en el que se encuentran las manufacturas en general, los productos agroalimentarios, productos químicos así como minerales y productos metálicos.

De cara al futuro las perspectivas son de un mantenimiento de esta tendencias, precisamente por el cariz de la internacionalización de los intercambios comerciales a los que ya se ha hecho referencia. En concreto "las nuevas y más modernas estructuras de intercambio, el establecimiento de redes, las relaciones bilaterales, los conceptos logísticos y la disminución de los gastos de transporte por unidad de producto se reflejan en la creciente complejidad, la intensificación y la dispersión en aumento de las estructuras de transporte en Europa, lo que favorece la posición de la carretera en el transporte de mercancías". Este vaticinio proviene nuevamente del documento de Noordwijk (pág. 14 y 15).

\footnotetext{
13 En el citado informe se dan detalles muy interesantes de lo que está lejos de parecer un mercado sin futuro (Transportes Ferroviarios Especiales S.A., Informe anual 1997). En el caso del Reino Unido durante 1997 destacan las 21.123 expediciones, cuatro trenes diarios, de piezas y componentes para la compañía Ford entre sus factorías de Almusafes en Valencia y Dagenham, Londres. Para dar una idea comparativa con los datos del cuadro 1 señalaremos además que este tráfico concreto alcanzó las 178.119 toneladas en 1996 y 189.967 en 1997, procediendo de la factoría británica algo más de la mitad del tráfico en las dos ocasiones.

${ }^{14}$ Nuestra fuente es la ya citada estadística sobre comercio exterior por modo de transporte que desde hace 15 años recopila y publica Eurostat, ya que entre otras cosas permite excluir el que se realiza entre los dos países ibéricos (que en especial para Portugal, y en determinados productos, representa una parte considerable de su transporte internacional total). Los datos referidos a la carretera tampoco están exentos de problemas, no sólo por haberse detectado errores sino por haber experimentado cambios en el sistema de recogida de información, basado hasta 1993 en las declaraciones de aduana y desde entonces en el sistema Intrastat. Como se sabe con este sistema se obliga a las empresas que mantengan relación comercial con los estados miembros de la Unión Europea, a presentar mensualmente una declaración sobre sus movimientos (importaciónexportación) de mercancías.
} 
Tabla 2.- Transporte de mercancías por carretera entre España y los demás estados ultrapirenaicos de la Union Europea (miles de toneladas).

\begin{tabular}{|lrrrrrr|}
\hline & \multicolumn{3}{c}{ Exportaciones* $^{*}$} & \multicolumn{3}{c|}{ Importaciones $^{*}$} \\
Destino/Origen & 1986 & 1995 & $\% 95 / 86$ & 1986 & 1995 & $\% 95 / 86$ \\
\hline Alemania & 1.158 & 305 & -74 & 1.180 & 1.540 & 31 \\
Francia & 4.500 & 9.440 & 110 & 3.495 & 7.842 & 124 \\
Italia & 445 & 1.088 & 144 & 290 & 1.190 & 310 \\
Países Bajos & 392 & 960 & 145 & 568 & 860 & 51 \\
Bélgica & 317 & 1.172 & 270 & 433 & 813 & 88 \\
Luxemburgo & 3 & 58 & 1.833 & 11 & 41 & 273 \\
Reino Unido & 598 & 663 & 11 & 386 & 1.068 & 177 \\
Irlanda & 17 & 22 & 29 & 4 & & \\
Dinamarca & 63 & 197 & 213 & 50 & 210 & 320 \\
Grecia & & 4 & & 17 & 16 & -6 \\
\hline
\end{tabular}

* Las casillas en blanco lo están por falta de datos en la fuente, que tampoco indica detalles del transporte con origen o destino en Austria, Suecia y Finlandia.

Fuente: Eurostat, El comercio exterior por modos de transporte, varios años. Elaboración propia.

Tabla 3.- Transporte de mercancías por carretera entre Portugal y los demás estados ultrapirenaicos de la Unión Europea (miles de toneladas).

\begin{tabular}{|lrrrrrrr|}
\hline & \multicolumn{3}{c}{ Exportaciones* $^{*}$} & \multicolumn{3}{c|}{ Importaciones $^{*}$} \\
Destino/Origen & 1986 & 1995 & $\% 95 / 86$ & 1986 & 1995 & $\% 95 / 86$ \\
\hline Alemania & 105 & 244 & 132 & 167 & 288 & 72 \\
Francia & 241 & 791 & 228 & 231 & 662 & 187 \\
Italia & 43 & 322 & 649 & 53 & 575 & 985 \\
Países Bajos & 36 & 118 & 228 & 52 & 112 & 115 \\
Bélgica & 28 & 96 & 243 & 44 & 43 & -2 \\
Luxemburgo & 1 & 14 & & 1 & 16 & \\
Reino Unido & 23 & 174 & 656 & 29 & 132 & 355 \\
Dinamarca & 9 & 44 & 388 & 12 & 45 & 275 \\
Suecia & & 26 & & & 3 & \\
Austria & & 23 & & & 13 & \\
\hline
\end{tabular}

${ }^{*}$ Las casillas sin datos tampoco los tienen en la fuente, que no incluye información sobre el transporte hacia/desde Irlanda, Grecia y Finlandia.

Fuente y elaboración, idem Tabla 2. 
Tabla 4.- Principales sectores en las importaciones y exportaciones transpirenaicas por carretera de España y Portugal en 1995 (miles de toneladas).

\begin{tabular}{|lcccc|}
\hline & \multicolumn{2}{c}{ España } & \multicolumn{2}{c|}{ Portugal } \\
Sector* & Exportaciones & Importaciones & Exportaciones & Importaciones \\
\hline 00 & 2.288 & 4.038 & 134 & 84 \\
01 & 1.810 & 1.126 & 214 & 173 \\
04 & 1.024 & 209 & 0 & 0 \\
05 & 1.118 & 724 & 137 & 21 \\
06 & 583 & 1.350 & 55 & 214 \\
07 & 99 & 400 & 0 & 0 \\
08 & 1.544 & 1.327 & 200 & 375 \\
09 & 5.321 & 4.100 & 1.104 & 1.017 \\
\hline
\end{tabular}

* Sectores: 00: productos agrícolas y animales vivos; 01: Productos alimentarios y piensos; 04: Minerales y chatarra; 05: Productos metálicos; 06: Minerales en bruto y materiales de construcción; 07: Fertlizantes; 08: Productos químicos; 09: Maquinaria, equipos de transporte, manufacturas diversas.

Fuente y elaboración: idem Tabla 2.

\section{EL INTENSO TRÁFICO DE VEHÍCULOS PESADOS POR LOS CARRETERAS Y AUTOPISTAS TRANSPIRENAICAS}

Las anteriores previsiones respecto del transporte por carretera en la Unión europea sólo suscitan preocupación habida cuenta de la naturaleza de sus secuelas, como las que se señalan en el citado docuemento de Nordwijk: crecimiento de la saturación y de las pérdidas de tiempo en algunas rutas, presión a favor de la realización de nuevas infraestructuras, emisiones de $\mathrm{CO}_{2}$ y de $\mathrm{NO}_{2}$, fragmentación de los paisajes, elevación de los niveles de contaminación acústica y otros diversos problemas medioambientales (pág. 27). Lo cierto es que dichas secuelas son ya una realidad en las carreteras transpirenaicas y su entorno, como consecuencia de la intensidad alcanzada por el paso de vehículos pesados y que se refleja en los datos del fichero de la Dirección General de Tráfico.

Precisemos primero que esta fuente clasifica los vehículos que pasan por las fronteras pirenaicas en tres categorías según su longitud: "cortos", "medianos" y "largos", y que dada la naturaleza de esta investigación -el transporte de mercancíasel análisis lo hemos centrado en la última categoría. Llamaremos pues vehículos pesados a lo que en la fuente aparece como "largos", y al igual que ocurre en fuentes 
similares ${ }^{15}$, los valores se refieren a todo tipo de vehículos largos, o pesados, incluídos autobuses de viajeros, que pueden tener una incidencia estacional muy importante en el tráfico ${ }^{16}$. Como ya se ha dicho los datos que aportamos corresponden al año $1997^{17}$ y se presentan en la Tabla 6, que detalla el promedio diario de las entradas y salidas de vehículos "largos" ("pesados" en el epígrafe) por 13 estaciones de toma de datos situadas en la misma raya fronteriza en otras tantas carreteras y autopistas transpirenaicas.

Tabla 6.- Tráfico de vehículos pesados a la entrada y la salida por las fronteras pirenaicas durante 1997 (vehículos/día).

\begin{tabular}{|lcc|}
\hline Estación de toma de datos & Entradas & Salidas \\
\hline Port-Bou (N-260) & 2,7 & 5,1 \\
La Junquera (A-7) & $2.906,0$ & $2.166,0$ \\
La Junquera (N-2) & 38,7 & 50,6 \\
Puigcerdá-Bourg-Madame & 73,2 & 92,9 \\
Seo de Urgell (N-145) & 251,9 & 152,3 \\
Les (N-230) & 110,2 & 99,1 \\
Canfranc (N-330) & 14,2 & 23,7 \\
Valcarlos-Pecocheta (N-135) & 19,2 & 22,0 \\
Dancharinea (N-121-B) & 19,2 & 24,4 \\
Behovia (A-8) & $2.147,9$ & $1.934,6$ \\
Behovia (N-121-A) & 301,8 & 345,8 \\
Irún, Pte. de Santiago (N-1) & 263,3 & 288,4 \\
\hline
\end{tabular}

Fuente: Fichero de la Dirección General de Tráfico. Elaboración Propia.

15 Nos referimos a los mapas de tráfico publicados por el ahora Ministerio de Fomento y que pueden servir también como aproximación al tráfico transpirenaico, aunque no puedan considerarse indicativos de entradas y salidas de vehículos dado que las estaciones de aforo no están en los puntos fronterizos sino en localidades cercanas.

${ }^{16}$ Esta es una cuestión esencial ya que es frecuente encontrar argumentaciones sobre el transporte de mercancías basadas en los datos publicados en mapas de tráfico, sin que se tenga en cuenta que la categoría de vehículos pesados es bastante heterogénea. En realidad hoy por hoy es imposible distinguir entre los diversos tipos de vehículos pesados, ya que su cómputo se basa, como se ha dicho, en la longitud que es lo que registran los sistemas electrónicos emplazados en las distintas estaciones de aforo. Sólo en una explotación ulterior de los datos, aplicándoles coeficientes derivados de observaciones sobre el terreno, es posible discriminar los distintos tipos de vehículos. Es lo que hace la estadística de Movimientos turísticos en fronteras (Frontur) a la que después nos referiremos.

17 Además de la falta de tiempo, hay otros motivos que explican que nos limitemos al año 1997. Es el más reciente y además el que se ha visto menos afectado en la toma y transmisión de datos por las numerosas contingencias que pueden alterar el funcionamiento de un sistema que, entre otras cosas, opera a la intemperie: falta de suministro de corriente, nevadas, deficiencias en el mantenimiento, huelgas de transporte, bloqueos en frontera, cambio de localización del sistema, etc. 

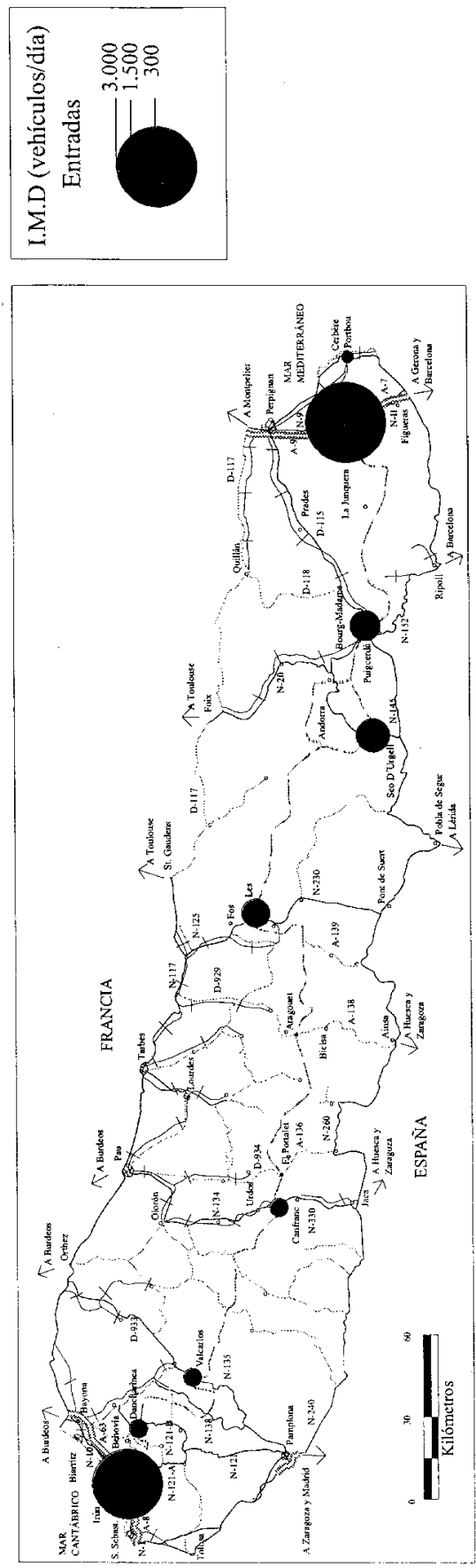
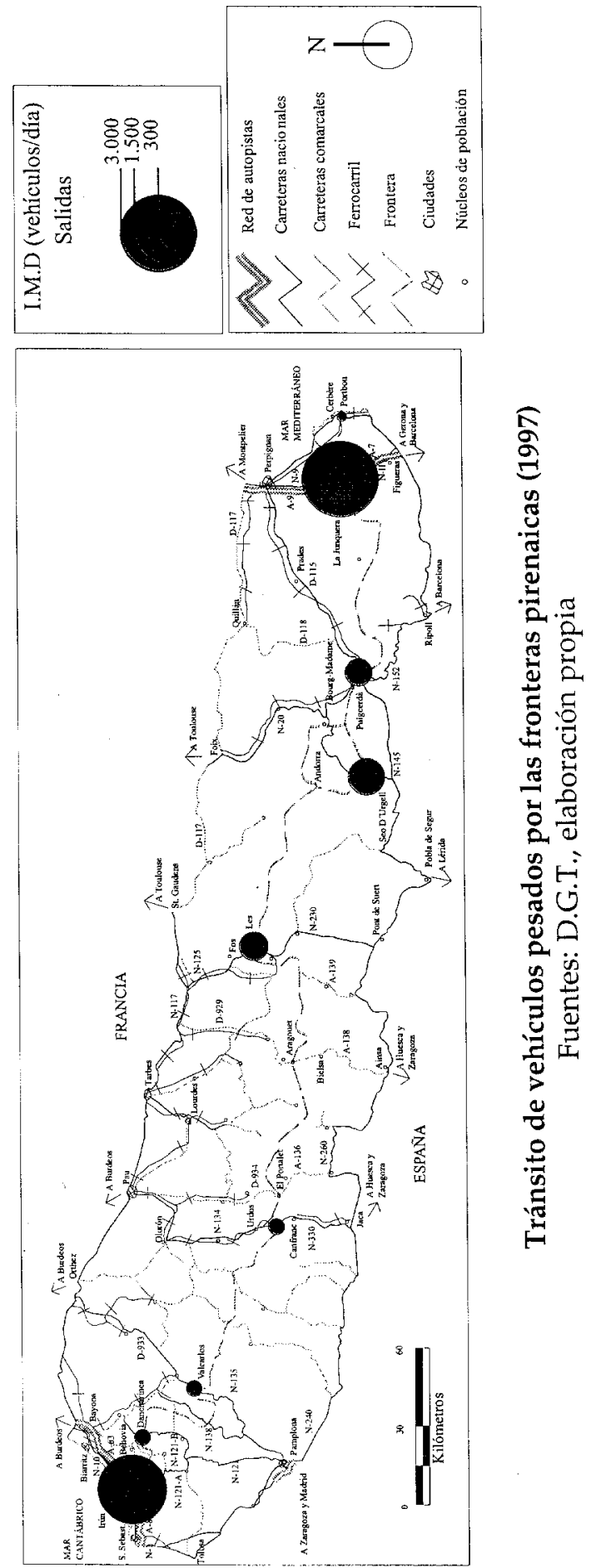
La Tabla 6 y los dos mapas de esferas adjuntos muestran una imagen del reparto del tránsito transpirenaico de vehículos pesados bastante expresiva y acorde con las consideraciones realizadas anteriormente. Por una parte queda evidenciada la jerarquización característica de la circulación transpirenaica, con su llamativo contraste entre el tráfico diario de las autopistas y carreteras nacionales de los dos corredores costeros, y el del resto de las travesías ${ }^{18}$. Sin duda alguna las mejores condiciones de capacidad, velocidad y servicio de dichos corredores son esenciales para obtener economías de escala y ventajas competitivas en un transporte, de mercancías y también de viajeros, predominantemente por carretera y a larga distancia, y en el que las regiones más avanzadas del nordeste y mediterráneo español tienen desde siempre gran protagonismo. Los mismos argumentos, pero en un razonamiento inverso, sirven para explicar la menor intensidad de circulación de vehículos pesados por las travesías del centro de los Pirineos.

El detalle con el que se presenta la información original nos permite añadir otras consideraciones sobre las características de la circulación transpirenaica de mercancías, como son su estacionalidad, con notables contrastes de circulación entre el mes de más tráfico de vehículos pesados y el de menos ${ }^{19}$; y su asimetría, con tráficos más intensos a la entrada, en algunas ocasiones, o a la salida. A este respecto véase cómo el tráfico de entrada está más concentrado en las autopistas y el de salida está más repartido. De ahí otra de las peculiaridades de la circulación por las carreteras centropirenaicas, como es que conocen un tránsito de vehículos pesados que da -con la excepción del valle de Arán- valores más altos a la salida de España que a la entrada.

Pero hay otra peculiaridad que, aunque no se refleja en esta fuente, no queremos dejar de resaltar, y es la alta proporción de vehículos que transportan mercancías peligrosas por las carreteras centropirenaicas. No era algo desconocido, pero queda palmariamente demostrado en los datos que por primera vez también publica el Ministerio de Fomento en su mapa de tráfico de 1996. Según dichos datos el tránsito medio diario de vehículos con mercancías peligrosas fue: 24 por La Junquera (N-2), 19 también por La Junquera (A-7), 13 en Puigcerdá, 11 en Les, 26 en Canfranc, 66 en Irún (N1) y 269 en Behovia (A-8). Puede observarse como en términos relativos, y con la excepción de la autopista A-8 y su gemela la $\mathrm{N}-1$, este tipo de transporte afecta de

\footnotetext{
${ }^{18}$ Hay que señalar además, según se nos comunicó en el centro de gestión de tráfico, la existencia de un fallo de calibraje del sistema electrónico en la estación de toma de datos de la carretera nacional-2, que se habría saldado con un subregistro sistemático de la categoría de vehículos "largos" por incluirse una parte en la de "medianos".

19 Por poner un ejemplo en la estación de toma de datos de la autopista A-7 el mayor número de vehículos pesados entró en el mes de julio (105.519) seguido de octubre (101.079); el de menos, por lo que a entradas se refiere, fue agosto con 66.025. En cuanto a tráfico diario, la mayor intensidad de entradas se registró el 31 de octubre con 5.083 vehículos.
} 
manera especial a las travesías por el centro de la cadena, lo que está en franca contradicción con la aspiración a un transporte sostenible por esta zona pirenaica ${ }^{20}$.

\section{ESPECIAL REFERENCIA AL TRÁFICO DE VEHÍCULOS PESADOS, Y CAMIONES EN PARTICULAR, A LA ENTRADA Y SALIDA POR EL PUERTO DE SOMPORT}

El análisis de la situación particular de la carretera del puerto de Somport presenta un interés especial, dadas las expectativas de crecimiento del tráfico de mercancías para cuando entre en servicio el túnel internacional. Por ello hemos confeccionado la tabla 7 con detalles de las entradas y salidas observadas en los meses del año en los que la estación suministró información. Los datos confirman que se trata de una ruta preferentemente de salida. de vehículos pesados, así como un importante factor estacional en la distribución mensual del tráfico, donde destacan claramente los meses de septiembre, octubre y noviembre. También fue de octubre el día de mayor número de salidas de vehículos, registrándose 56 el día 30.

Tabla 7.- Entradas y salidas de vehículos pesados por la frontera de Canfranc durante 1997.

\begin{tabular}{|lcccc|}
\hline & \multicolumn{2}{c}{ Entradas } & \multicolumn{2}{c|}{ Salidas } \\
Mes* & Vehículos & $\mathrm{N}^{\text {o }}$ de días & Vehículos & $\mathrm{N}^{0}$ de días \\
\hline Enero & s.d. & & 452 & 23 \\
Febrero & s.d. & & 217 & 11 \\
Marzo & s.d. & & s.d. & \\
Abril & 71 & 4 & 408 & 18 \\
Mayo & 435 & 28 & 469 & 28 \\
Junio & 368 & 26 & 363 & 26 \\
Julio & 212 & 29 & 527 & 29 \\
Agosto & 168 & 20 & 561 & 31 \\
Septiembre & 488 & 30 & 969 & 30 \\
Octubre & 562 & 31 & 1.018 & 31 \\
Noviembre & 376 & 27 & 682 & 30 \\
Diciembre & 192 & 31 & 500 & 31 \\
\hline
\end{tabular}

s.d., sin datos. Fuente y elaboración: idem Tabla 6.

${ }^{20}$ Véase, entre otros, Etchélecou, A., Axe Pau-Somport. Quel trafic de poids lourds? Le prévu et le probable. IRSAM, Universidad de Pau, 1995. En dicho estudio se publican los resultados de observaciones del paso de vehículos con estas características por el valle de Aspe, lo que permitió identificar hasta 24 productos peligrosos distintos. 
La Tabla anterior presenta lagunas en el cómputo de las entradas de vehículos por Canfranc durante el primer trimestre del año. Por ello hemos buscado completar 1 a serie recurriendo a otra fuente gemela, como es la estadística de Movimiento Turístico en Fronteras (Frontur) que, para sus propios fines, obtiene información en frontera sobre los distintos tipos de vehículos que entran en España ${ }^{21}$. Son datos referidos únicamente a entradas, pero no por ello menos expresivos, y que se saldan para 1997 con un sustancial incremento del promedio diario de entradas de vehículos pesados que, según esta fuente, habría sido en realidad de $23,8^{22}$.

Conviene seguir recordando, en cualquier caso, que los datos que hemos manejado hasta ahora incluyen vehículos ajenos al tránsito de mercancías. Ello no es necesariamente malo para nuestros fines ya que refleja la intensidad que puede alcanzar la circulación cuando coinciden circunstancias favorables para el tránsito masivo de personas y de mercancías. Esas mismas rutas, por supuesto, conocen a su vez una mayor o menor frecuentación por vehículos automóviles. No obstante para insistir en el cariz poco sostenible del transporte actual de mercancías a través del Pirineo central, y por el puerto de Somport en particular, resulta indispensable contar con información específica relativa al tráfico de camiones. De ahí el interés de la ya citada estadística "Movimiento Turístico en Fronteras" y, en concreto, la tabla TA.E.75 ("Número de vehículos contabilizados a la entrada en España, por paso fronterizo, según medio de transporte"). De la misma proceden los datos de la Tabla 8 según los cuales, en la línea de lo ya sabido, la autopista A-7 y muy por detrás la A-8, se confirman como las vías de entrada de camiones en nuestro país. Proporciones variable entre el 0,3 y el $0,6 \%$ del total de entradas corresponden a las demás carreteras transpirenaicas. Ahora bien, lo que hemos llemado en la Tabla "\% horizontal" -indicador de la proporción de camiones en el total de entradas de vehículos pesados por cada carretera -, acerca notablemente posiciones entre unas y otras; sitúa -desde este punto de vista- a la autopista del Pais Vasco, la A-8, por delante de la del Mediterráneo, A-7; y muestra que también en las travesías centropirenaicas los camiones representan más del $50 \%$ de sus tráficos respectivos de vehículos pesados, dándose la circunstancia de que la N-230, que recorre los parajes del valle de Aran, fue la que alcanzó la proporción más alta de todo el conjunto.

${ }^{21} \mathrm{El}$ interés de esta fuente es que utiliza la información generada por la Dirección General de Tráfico, que hemos presentado en el punto anterior, pero la depura y -en realidad- mejora, a partir de un minucioso trabajo de campo. De este modo se obtiene una estadística que, aunque limitada a las entradas de.vehículos, resulta de gran utilidad para fines diversos, por lo que ha recibido un notable reconocimiento en todo el mundo (Instituto de Estudios Turísticos, Informe anual del Movimiento Turístico en Fronteras, año 1997).

22 Efectivamente, como se detalla en la base de datos a la que henos tenido acceso, los encuestadores contratados por el Instituto de Turismo contaron 1.075 vehículos pesados en los 45 días que estuvieron apostados en la frontera de Canfranc durante 1997 (repartidos entre 4 y 6 días por mes con la excepción de febrero y julio de los que no hay datos). 
Tabla 8.- Proporción de camiones en las entradas de vehículos por los pasos fronterizos con Francia durante 1997.

\begin{tabular}{|lccc|}
\hline & & \multicolumn{3}{c|}{$\%$ horizontal } \\
Paso fronterizo & \% vertical & A & B \\
\hline Puente de Santiago (N-10) & 3,5 & 9,3 & 66 \\
Behovia (N-121-A) & 3,5 & 10,6 & 65,2 \\
Biriatou (A-8) & 28,8 & 29,6 & 75,2 \\
Dancharinea (N-121-B) & 0,6 & 5,7 & 60,3 \\
Valcarlos (N-135) & 0,3 & 8,1 & 56,4 \\
Canfranc (N-330) & 0,4 & 10,1 & 50,2 \\
Les (N-230) & 0,5 & 8,6 & 80,1 \\
Seo de Urgell (N-145) & 5,1 & 4,8 & 56,6 \\
Puigcerdá-Bourg-Madame (N-152) & 0,5 & 4,2 & 55,3 \\
La Junquera (N-2) & 0,4 & 3 & 30,6 \\
La Junquera (A-7) & 43,3 & 26,5 & 70,8 \\
Port Bou (N-260) & 0,2 & 3,4 & 32,2 \\
\hline
\end{tabular}

A) \% camiones/total vehículos; B) \% camiones/vehículos pesados.

Fuente: Movimientos Turísticos en Fronteras (Frontur), 1997. Elaboración propia.

\section{CONCLUSIONES}

Como se apuntaba en la introducción, el objetivo de este artículo era mostrar la aplicación de dos fuentes recientes al análisis del transporte de mercancías a través de los Pirineos, y paliar así el déficit informativo suscitado por el cierre de las aduanas pirenaicas. Las posibilidades de las dos fuentes son mucho mayores dado su nivel de detalle y su rigor. No obstante, a la espera de completar su explotación, esperamos haber cumplido con el otro cometido de este trabajo, como es el de confirmar los planteamientos de quienes demandan cambios en la organización de la circulación transpirenaica de mercancías dada la intensidad actual y las previsiones a corto y medio plazo. Las alternativas que se manejan, a favor del ferrocarril y del centro de la cadena, son bien conocidas y se amparan en una abundantísima documentación que pone de manifiesto la inviabilidad de los patrones de circulación de mercancías vigentes en Europa, y ello por motivos medioambientales, de disponibilidad de espacio y de ordenación del territorio. Sobran, pués, las razones. ¿Será suficiente? 
